\title{
Proteína C-reactiva asociada a la condición periodontal de gestantes
}

Sandra Jessenia Pesantes Sangay 1,a ; Pablo Alejandro Millones Gómez* 2,b

\section{RESUMEN}

Objetivo: Determinar el nivel de proteína C-reactiva según el estado periodontal, edad y trimestre de gestación en gestantes del Centro de Salud Magna Vallejo en Cajamarca, Perú.

Materiales y métodos: Estudio observacional, descriptivo y transversal. Se trabajó con una muestra de 60 gestantes divididas en tres grupos: gestantes periodontalmente sanas, con gingivitis y con periodontitis. Se empleó el índice de Rusell para la determinación del estado periodontal. El valor del nivel de proteína C-reactiva fue tomado del registro de la historia clínica de cada paciente.

Resultados: Se encontró un nivel de proteína C-reactiva elevado en el 66,70\% de gestantes con gingivitis y en el $100 \%$ de gestantes con periodontitis, así mismo en el $84,60 \%$ de gestantes adultas.

Conclusiones: Los niveles de proteína C-reactiva tienden a elevarse en las pacientes con enfermedad periodontal y se incrementa con la edad de las gestantes.

Palabras clave: Proteína C-reactiva; Gingivitis; Periodontitis (Fuente: DeCS BIREME).

\section{C-reactive protein associated with the periodontal condition of pregnant women}

\section{ABSTRACT}

Objective: To determine the C-reactive protein level according to the periodontal state, age and trimester of pregnancy in pregnant women who attend the Centro de Salud Magna Vallejo, Cajamarca, Peru.

Materials and methods: An observational, descriptive and cross-sectional study conducted in a sample of 60 pregnant women divided into three groups: those who were periodontally healthy, those with gingivitis and those with periodontitis. The Russell Index was used to determine the periodontal status. The value of the C-reactive protein level was taken from each patient's medical record.

Results: An elevated C-reactive protein level was found in $66.70 \%$ of the pregnant women with gingivitis, $100 \%$ of the pregnant women with periodontitis and $84.60 \%$ of the adult pregnant women.

Conclusions: C-reactive protein levels tend to rise in patients with periodontal disease and increase in adult pregnant women.

Keywords: C-Reactive protein; Gingivitis; Periodontitis (Source: MeSH NLM).

\footnotetext{
1 Universidad César Vallejo. Escuela de Estomatología. Piura, Perú.

2 Universidad Privada de Huancayo Franklin Roosevelt, Escuela de Estomatología. Huancayo, Perú.

a Maestra en Estomatología, Cirujano dentista especialista en Periodoncia.

b Doctor en Estomatología, Cirujano dentista especialista en Periodoncia.

* Autor corresponsal
} 


\section{INTRODUCCIÓN}

La relación bilateral entre la enfermedad periodontal y el embarazo es bien conocida desde hace muchos años, y se confirma un aumento de la incidencia en esta etapa ${ }^{(1,2)}$. Asimismo, son conocidos los efectos de la enfermedad periodontal sobre la gestación, como parto pretérmino, retardo del crecimiento intrauterino, bajo peso al nacer y preeclampsia ${ }^{(3,4)}$, complicaciones que representan un problema de salud pública de significancia mundial, ya que constituyen las principales causas de mortalidad materna y neonatal ${ }^{(3)}$.

La evidencia científica nos indica que, durante la enfermedad periodontal, se incrementa la concentración de marcadores inflamatorios, entre ellos la proteína C-reactiva ${ }^{(5,6)}$, cuya identificación podría ser de utilidad en el establecimiento de la magnitud de la enfermedad periodontal y posibles factores de riesgo durante la gestación.

La proteína C-reactiva es un reactante de fase aguda sintetizado en el hígado y su incremento está regulado por el aumento de citoquinas proinflamatorias, en especial, la interleucina 6 (IL-6) y en menor frecuencia, la interleucina 1 (IL-1) y el factor de necrosis tumoral a (TNFa, por sus siglas en inglés) que responden al daño inducido en los tejidos por inflamaciones, infecciones o neoplasias ${ }^{(6-9)}$.

Se ha descrito que 6 a 12 horas después de empezar el estímulo inflamatorio, la proteína C-reactiva se incrementa y alcanza sus valores máximos entre las 24 a 72 horas, con una vida media de alrededor de 19 horas ${ }^{(8,9)}$. Acabado el estímulo, sus valores se normalizan a los 7 días, aproximadamente. Su índice de producción establece sus valores circulantes, reflejando la intensidad de los procesos patológicos que contribuyeron a su estímulo ${ }^{(9)}$.

La proteína C-reactiva se liga a la fosfocolina y reconoce patógenos externos; además, inhibe la adhesión leucocitaria al endotelio y la generación de superóxidos en los neutrófilos y estimula la síntesis de factor tisular en los monocitos y citocinas inflamatorias ${ }^{(10)}$.

Se ha determinado que la proteína C-reactiva no se comporta solamente como un marcador inflamatorio sistémico, sino que tiene efectos proinflamatorios que incrementan la lesión en los tejidos y conducen a una enfermedad más grave. Este hecho la convierte en un participante directo en distintos cuadros en los que interviene la respuesta inmune innata ${ }^{(7)}$.

Diferentes mecanismos de muerte celular programada (apoptosis) e inflamaciones subclínicas van incrementándose conforme pasan los años, y la edad puede influir en el aumento del nivel de proteína C-reactiva (7). Así mismo, la edad gestacional podría ocasionar una variación en los niveles de proteína C: se han encontrado reportes de gestantes del segundo y tercer trimestre con niveles séricos más elevados lo que se asocia a riesgo de parto pretérmino, bajo peso al nacer y preeclampsia ${ }^{(11-14)}$.

Las lesiones inflamatorias menores pueden incrementar los valores de proteína C-reactiva, por lo que es un marcador altamente sensible, y se convierte en una medida fiable para la evaluación y el seguimiento de la inflamación ${ }^{(10)}$, como en el caso de la enfermedad periodontal en el embarazo.

Durante el embarazo, la inmunosensibilidady los mediadores de la respuesta inflamatoria se ven alterados debido al aumento de los niveles de estrógenos y progesterona. Los cambios en los valores hormonales, así como en los hábitos de higiene oral y estilo de vida, pueden dar lugar a una mayor susceptibilidad de las mujeres embarazadas a la enfermedad periodontal ${ }^{(3,15)}$.

Los diversos estudios que han evaluado la asociación entre la enfermedad periodontal y marcadores inflamatorios como la proteína C-reactiva han encontrado un aumento progresivo de sus niveles, según la severidad de la enfermedad periodontal (16-22); asimismo, se evidencia una disminución de dichos niveles después de que los pacientes fueron sometidos a tratamiento periodontal $(6,16,23)$. Sin embargo, existen muy pocas investigaciones en gestantes, lo que merecería su continuo estudio, ya que tal asociación no solo involucra el hallazgo de un biomarcador inflamatorio, sino de un factor de riesgo. Estudios recientes confirman que los niveles altos de proteína C-reactiva estimulan la producción de citoquinas inflamatorias, la activación del complemento y el daño en los tejidos, lo que incrementa la respuesta inflamatoria ${ }^{(5,7,10)}$, que es considerada patogénica en el desarrollo de algunas enfermedades como la hipertensión arterial (7), lo que incrementaría el riesgo de eventos adversos en el embarazo ${ }^{(24-26)}$.

Según los últimos datos estadísticos del Banco Mundial, la tasa de mortalidad materna en el Perú asciende a 88 por cada 100000 nacidos vivos ${ }^{(27)}$, una de sus principales causas es la hipertensión gestacional (preeclampsia y eclampsia). Por otra parte, la tasa de mortalidad neonatal se estima en 7,3 por cada 1000 nacidos vivos ${ }^{(27)}$, sus causas son el parto prematuro y el bajo peso del bebé al nacer, la etiología de ambos, a su vez, se relacionaría la hipertensión gestacional. En consecuencia, la interpretación que el personal médico, incluido el equipo odontológico, le da a este biomarcador inflamatorio en los diversos entornos clínicos de la gestante como condiciones estomatológicas y periodontales, se vuelve importante. De la misma manera en que la edad de la gestante y la edad gestacional evidencian cambios anatómicos y fisiológicos diferentes, también se observan situaciones clínicas periodontales distintas, lo que ameritaría un estudio minucioso de los niveles de proteína C-reactiva en dichos contextos, que darían oportunidades para la prevención y el 
manejo temprano de enfermedades periodontales en esta esta etapa de vida tan susceptible de la mujer.

El presente estudio tuvo como propósito determinar el nivel de proteína C-reactiva según estado periodontal, edad y trimestre de gestación, en gestantes atendidas en el Centro de Salud Magna Vallejo.

\section{MATERIALES Y MÉTODOS}

\section{Diseño y población de estudio}

La presente investigación es de tipo observacional, descriptiva y de corte transversal, y fue realizada en el Centro de Salud Magna Vallejo, Cajamarca - Perú.

La población estimada, basada en los reportes del servicio de odontología y que cumplió con los criterios de inclusión, fue de 150 gestantes. El cálculo del tamaño de la muestra se determinó mediante la fórmula estadística para la estimación de una proporción poblacional, más la fórmula para muestras reajustadas ${ }^{(28)}$. Finalmente, la muestra estuvo conformada por 60 gestantes seleccionadas por muestreo sistemático aleatorio simple. Los criterios de inclusión que se establecieron fueron los siguientes: gestantes $\geq 18$ años, con al menos 20 piezas dentales, en aparente buen estado de salud sistémico, nutricional y mental, lúcidas y orientadas en tiempo, espacio y persona. Se excluyeron aquellas gestantes que ingerían de algún tipo de fármaco sistémico y que consumían de cigarrillos, alcohol y/o drogas.

\section{Variables y mediciones}

Las gestantes fueron distribuidas en 3 grupos, según el estado periodontal encontrado: gestantes periodontalmente sanas, gestantes con gingivitis y gestantes con periodontitis. Para el diagnóstico periodontal se empleó una sonda periodontal tipo OMS, espejos bucales sin aumento N. ${ }^{\circ} 5$ con mango y una fuente de luz artificial. Se aplicó el índice periodontal de Rusell (PI) ${ }^{(29)}$ con la siguiente clasificación: 0 = encía sana, sin evidencia de alteración; 1 = gingivitis leve: área de inflamación en la encía libre, no circunscribe el diente; 2 = gingivitis, con área de inflamación en toda la encía libre que rodea el diente, pero no existe ruptura evidente de la adherencia epitelial; 4 = usado cuando las radiografías son posibles de realizar. No hay valoración clínica, pequeña pérdida ósea a rayos X; 6 = gingivitis con formación de bolsa: ruptura de la adherencia epitelial y evidencia de bolsa periodontal. No hay interferencia con la masticación, ni existe movilidad dentaria; y 8 = destrucción avanzada con pérdida de la función masticatoria, bolsa profunda, movilidad evidente. Se consideraron los valores 1 y 2 como gingivitis y los valores 6 y 8 como periodontitis. El criterio 4 no fue tomado en cuenta para evitar la radiación en la gestante. El operador fue calibrado para el registro del índice periodontal de Rusell por un especialista en Periodoncia, para lo cual se realizó una calibración intra e inter observador y se empleó el índice de Kappa de Cohen, con lo que se obtuvo un nivel de concordancia muy bueno de 0,82 y 0,91 , respectivamente.

La duración del embarazo se registró por trimestres de gestación y la edad de la gestante se registró de la siguiente manera: gestante joven (si encontraba entre los 18 y 29 años de edad) y gestante adulta (si fue $\geq 30$ años).

Como protocolo del Centro de Salud, todas las gestantes nuevas que acuden a control deben someterse a análisis clínicos de rutina. El protocolo que manejó el laboratorio del establecimiento para la determinación in vitro del nivel de proteína C-reactiva fue el siguiente: de aquellas muestras recolectadas de las gestantes, previamente centrifugadas, se tomó 40 ul de suero sanguíneo y se empleó el test en placa mediante aglutinaciones, con el reactivo látex PCR directo de laboratorios QCA (técnica cualitativa) ${ }^{(30)}$, siguiendo las especificaciones técnicas del fabricante. Se colocó una gota del reactivo y se mezcló de manera circular con el suero sanguíneo hasta homogenizar la muestra y se esperó tres minutos para observar la reacción, si la mezcla se conservó uniforme entonces se reportó como "Negativo", lo que significa que el valor de la proteína C-reactiva se encontró dentro del nivel normal $\leq 7,5 \mathrm{mg} / \mathrm{L}$ (sin inflamación) y, si en ese lapso se observó aglutinación, se reportó como "Positivo", lo que indica que el valor de la proteína C-reactiva se encontró elevado, es decir $>7,5 \mathrm{mg} / \mathrm{L}$ (con inflamación). Los resultados de los análisis clínicos de proteína C-reactiva fueron registrados en la ficha de recolección de datos, elaborada para dicho fin, así como también los datos del estado periodontal, la edad de la gestante y el trimestre de gestación.

\section{Análisis estadístico}

Se empleó el programa estadístico SPSS Statistics 22,0 (IBM, Armonk, NY, EE. UU.) para el procesamiento de la información y se presentaron los resultados en tablas de doble entrada. Se calcularon las frecuencias absolutas y porcentuales del nivel de proteína C-reactiva según el estado periodontal, edad y trimestre de gestación. Para comparar el nivel de proteína C-reactiva, según las diferentes variables, se utilizó la prueba $Z$ de comparación de proporciones. Para determinar la asociación del estado periodontal, trimestre de gestación y la edad de la gestante sobre la proteína C-reactiva se emplearon modelos de regresión logística binaria. Se consideró un nivel de significancia del $5 \%$.

\section{Consideraciones éticas}

Para la ejecución del presente estudio se contó con la aprobación del jurado evaluador de la Unidad de Posgrado en Ciencias Médicas de la Universidad Nacional de Trujillo, la aprobación de la dirección de la Microrred de Salud Magna Vallejo y el consentimiento informado de la paciente. Se siguieron los principios éticos de la Declaración de Helsinki, 
de la Asociación Médica Mundial y las pautas éticas internacionales para investigación biomédica en seres humanos del Consejo de Organizaciones Internacionales de Ciencias Médicas (CIOMS).

\section{RESULTADOS}

Del total de gestantes evaluadas, 7 presentaron periodonto sano $(11,67 \%), 42$ mostraron gingivitis $(70 \%)$ y en 11 encontramos periodontitis (18,33\%). El nivel de proteína C-reactiva se mostró dentro de los valores normales en 21 gestantes evaluadas ( $35 \%$ ) y se encontró un valor elevado en las otras 39 (65\%). El estado periodontal se mantuvo dentro de los valores normales $(\leq 7,5 \mathrm{mg} / \mathrm{L})$ en las 7 gestantes con periodonto sano $(100 \%)$, se encontró elevado $(\geq 7,5 \mathrm{mg} / \mathrm{L}$ ) en 28 gestantes con gingivitis $(66,70 \%)$, y en las 11 gestantes con periodontitis (100\%).

El nivel de proteína C-reactiva, según la edad de la gestante, fue el siguiente: elevado en $20(52,60 \%)$ de las 38 gestantes jóvenes de 18 a 29 años; de igual manera, 19
$(86,40 \%)$ de las 22 gestantes adultas $\geq 30$ años de edad. Las demás participantes presentaron niveles normales. Según trimestre de gestación, el nivel de proteína C-reactiva fue elevado en 6 de las 13 gestantes evaluadas de primer trimestre $(46,20 \%)$, en 18 de las 28 gestantes de segundo trimestre $(64,30 \%)$ y en 15 de las 19 gestantes del tercer trimestre $(78,90 \%)$; las demás se mantuvieron dentro del nivel normal (Tabla 1).

Al determinar la asociación del nivel de proteína C-reactiva según estado periodontal, trimestre de gestación y edad de la gestante, se hallaron los siguientes resultados: el nivel de proteína C-reactiva evidenció diferencia estadística significativa entre los grupos normal y elevado en los pacientes con periodonto sano, gingivitis y periodontitis $(p<0,05)$; de igual manera entre los grupos normal y elevado en las gestantes adultas $(p<0,001)$ y también entre los grupos normal y elevado en las gestantes del tercer trimestre $(p<0,05)$ (Tabla 1).

Tabla 1. Comparación de proporciones del nivel de proteína C-reactiva en gestantes según estado periodontal, trimestre de gestación y edad de la gestante

\begin{tabular}{|c|c|c|c|c|c|}
\hline \multirow{2}{*}{ Variable } & \multirow{2}{*}{ Grupos } & \multirow{2}{*}{$\mathbf{N}$} & \multicolumn{2}{|c|}{ Nivel de PCR } & \multirow{2}{*}{$p^{*}$} \\
\hline & & & $\begin{array}{c}\text { Normal } \\
\text { n (\%) }\end{array}$ & $\begin{array}{c}\text { Elevado } \\
\text { n (\%) }\end{array}$ & \\
\hline \multirow{3}{*}{$\begin{array}{c}\text { Estado } \\
\text { periodontal }\end{array}$} & Sano & 7 & $7(100,0)$ & $0(0,0)$ & 0,001 \\
\hline & Gingivitis & 42 & $14(33,3)$ & $28(66,7)$ & 0,004 \\
\hline & Periodontitis & 11 & $0(0,0)$ & $11(100,0)$ & $<0,001$ \\
\hline \multirow{3}{*}{$\begin{array}{l}\text { Edad de la } \\
\text { gestante }\end{array}$} & Joven & 38 & $18(47,4)$ & $20(52,6)$ & 0,819 \\
\hline & Adulta & 22 & $3(13,6)$ & $19(86,4)$ & $<0,001$ \\
\hline & $1 .^{e r}$ & 13 & $7(53,8)$ & $6(46,2)$ & 1,000 \\
\hline \multirow[t]{2}{*}{$\begin{array}{l}\text { Trimestre de } \\
\text { gestación }\end{array}$} & 2. er & 28 & $10(35,7)$ & $18(64,3)$ & 0,060 \\
\hline & 3. er & 19 & $4(21,1)$ & $15(78,9)$ & 0,001 \\
\hline
\end{tabular}

En la determinación Odds-ratio se encontró diferencia $\mathrm{OR}=5,70($ Tabla 2). significativa en la edad de la gestante $(p<0,05)$ con un 
Tabla 2. Prueba de regresión logística para la determinación de la asociación del nivel de proteína C-reactiva en gestantes según estado periodontal, trimestre de gestación y edad de la gestante

\begin{tabular}{|ccccc}
\hline Variable & $\mathrm{n}$ & $\mathrm{B}$ & $\mathrm{OR}$ & $\mathrm{p}^{*}$ \\
\hline Estado periodontal & 60 & 21,21 & 1,63 & 0,998 \\
\hline Trimestre de gestación & 60 & 0,74 & 2,09 & 0,062 \\
\hline Edad de la gestante & 60 & 1,74 & 5,70 & 0,013 \\
\hline$p^{*}$ : Regresión logística & & & & \\
$\begin{array}{l}\text { B: Coeficiente del modelo } \\
\text { OR: Odds Ratio. }\end{array}$ & & & & \\
\hline
\end{tabular}

\section{DISCUSIÓN}

Es bien conocida la relación entre el embarazo y la enfermedad periodontal. La evidencia científica nos indica que durante el desarrollo de esta enfermedad se incrementa la concentración de marcadores inflamatorios, como la proteína C-reactiva ${ }^{(5,6)}$, por lo que su identificación es útil para determinar la magnitud de la enfermedad periodontal y los posibles factores de riesgo durante la gestación, ya que no solo se comporta como un biomarcador, sino que, además, tiene una participación activa en la inflamación, como lo mencionan Tang et al. ${ }^{(7)}$ y Sproston et al. ${ }^{(10)}$.

Observamos la presencia de la enfermedad periodontal en el $88,30 \%$ de gestantes evaluadas, porcentaje que se aproxima a los datos estadísticos de la Organización Panamericana de la Salud (OPS) que reporta un $85 \%$ de enfermedad periodontal en el Perú (31), lo que significa que representa uno de los problemas de salud bucal de mayor frecuencia en nuestra población. La gingivitis es una condición que se presenta en mayor porcentaje, y en nuestro estudio representa un $70 \%$ frente a la periodontitis que se observó solo en el 18,33 \% de los casos evaluados. En referencia al nivel de proteína C-reactiva de las gestantes evaluadas, se detectó la presencia de nivel elevado de proteína C-reactiva en el $65 \%$ de los casos evaluados, lo que nos indica que la proteína C-reactiva constituye un marcador inflamatorio de alta sensibilidad, lo que coincide con lo reportado por Sproston et al. (10), quienes señalan su incremento dramático en respuesta a injurias, infección e inflamación.

En cuanto al nivel de proteína C-reactiva según estado periodontal, las gestantes con gingivitis presentaron un valor elevado en la mayoría de los casos evaluados, y se evidencia una diferencia significativa en relación al grupo de casos que presentaron valores normales $(p<0,05)$. Asimismo, las gestantes con periodontitis mostraron un nivel de proteína C-reactiva elevado en todos los casos evaluados, con una diferencia estadística altamente significativa $(p<0,001)$, por lo que se evidencia un aumento del número de casos de proteína C-reactiva detectable en suero conforme avanza la enfermedad. Estos resultados coinciden con Martínez et al. ${ }^{(8)}$ y Mannava et al. ${ }^{(32)}$ que sostienen que al ser la proteína C-reactiva un reactante de fase aguda que se produce en respuesta al incremento de interleucinas inflamatorias, sus niveles pueden llegar a elevarse en presencia de la enfermedad periodontal. Además, se encuentra concordancia con los estudios Bansal et al. (17) y Goyal et al. (18) que reportaron niveles medios de proteína C-reactiva más altos en el grupo de periodontitis y plantearon que, con el aumento de la inflamación, los niveles se elevan proporcionalmente, esto debido, posiblemente, a la destrucción progresiva que causa esta enfermedad inflamatoria en el periodonto, lo que involucra la pérdida del tejido óseo. Por lo tanto, se comprende que exista un número mayor de casos con altos valores de proteína C-reactiva en este estado periodontal.

En cuanto a la edad de la gestante, en las gestantes adultas de 30 años a más se estableció un incremento del número de casos con alto valor de proteína C-reactiva con una diferencia estadística altamente significativa entre los grupos con nivel de proteína C-reactiva normal y elevado $(p<0,001)$, dato que coincide con el estudio de Wyczalkowska (33) que muestra que la edad guarda relación con el incremento de los niveles séricos de proteína C-reactiva, dada la mayor cantidad de procesos inflamatorios subclínicos. Dado que la condición inflamatoria del periodonto se incrementa a mayor presencia de biofilm bacteriano ${ }^{(34-37)}$ y que la gran mayoría de pacientes de nuestro estudio provienen de zona rural, viven en extrema pobreza y muchas de ellas no han sido concientizadas en prácticas adecuadas de higiene oral, o han recibido tratamientos odontológicos previos, se podría inferir que a mayor edad, mayor depósito de placa bacteriana y, por ende, mayor inflamación periodontal, por lo que se incrementaría el porcentaje de casos de proteína C-reactiva con nivel elevado.

Según el trimestre de gestación, se observó un incremento del porcentaje de casos de proteína C-reactiva con nivel 
elevado en el tercer trimestre de embarazo, se evidencia una diferencia estadística altamente significativa en comparación con el grupo que mantuvo sus niveles normales $(p<0,001)$, dato que coincide con los estudios de Ali et al. (13) y Rout et al. (14), esto debido a los cambios en el organismo de la gestante, principalmente en el útero, en las últimas semanas de gestación. Además, habría que considerar que la variación de la situación hormonal de la mujer y su repercusión en los tejidos periodontales proporcionan condiciones óptimas para la colonización de especies microbianas que, generalmente, no forman parte del microambiente periodontal y que inducen la destrucción del tejido a través de enzimas proteolíticas. $\mathrm{Si}$ consideramos que tales microorganismos forman biofilms que les permiten agruparse en "comunidades" organizadas y tenaces, es comprensible que desarrollen una resistencia a la respuesta inmune del huésped, la misma que, al no resolverse, se convierte en la principal responsable de la destrucción del periodonto, cuya condición inflamatoria se exacerba, conforme avanza la gestación, por la persistencia del factor hormonal y el mayor formación de biofilms en las zonas de profundidad ${ }^{(2,34-37)}$. Por todo ello, es evidente que en el último trimestre se encuentra el mayor porcentaje casos de nivel elevado de proteína C-reactiva.

En la determinación de la asociación del estado periodontal, el trimestre de gestación y la edad de la gestante sobre el nivel de la proteína C-reactiva, se pudo observar que solo la edad de la gestante presentó una diferencia significativa alta con un $O R=5,70$, lo que evidencia que las gestantes adultas presentan 4,70 veces más probabilidad de mostrar valor elevado de proteína C-reactiva, lo que puede, a su vez, aumentar la respuesta inflamatoria, y convertirse en un factor de riesgo predictor para resultados adversos en el embarazo, por lo que el conocimiento de sus niveles en diferentes etapas de vida, en especial en la edad adulta, permitiría establecer medidas de prevención que mejoren la calidad de vida de las gestantes y del bebé por nacer.

Se puede concluir que los niveles de proteína C-reactiva tienden a elevarse en las pacientes con enfermedad periodontal, hecho que se incrementa con la edad de las gestantes.

Contribuciones de los autores: SJPS ha participado en la concepción y diseño del estudio, recolección de datos, obtención de resultados, análisis e interpretación de los datos y redacción del informe. PAMG en la revisión crítica del manuscrito, asesoría técnica o administrativa, y aprobación de versión final informe.

Fuentes de financiamiento: Este artículo ha sido financiado por los autores.

Conflictos de interés: Los autores declaran no tener ningún conflicto de interés.

\section{REFERENCIAS BIBLIOGRÁFICAS}

1. Rashidi F, Haerian A, Vaziri F, Khabbazian A, Mohammadi S. CPITN changes during pregnancy and maternal demographic factors' impact on periodontal health. Iran J Reprod Med. 2015; 13(2): 107-12.

2. Lu H, Xu W, Wei T, Feng X. Impact of periodontal conditions on the quality of life of pregnant women: a cross-sectional study. Health Qual Life Outcomes. 2015; 13: 67.

3. Gesase N, Miranda-Rius J, Brunet-Llobet L, Lahor-Soler E, Mahande MJ, Masenga G. The association between periodontal disease and adverse pregnancy outcomes in Northern Tanzania: a cross-sectional study. Afr Health Sci. 2018; 18(3): 601-11.

4. Makeeva I, Ignatko A, Churganova A, Lebedev V, Makeeva M. Periodontal diseases and complicated pregnancy. Stomatologiia (Mosk). 2019; 98(1): 7-73.

5. Ersin R, Öngöz F, Gökmenoğlu C, Kara C. Salivary fetuin-A, S100A12, and high-sensitivity C-reactive protein levels in periodontal diseases. Oral Dis. 2018; 24(8): 1554-61.

6. Ahmad A, Nazar Z, Swaminathan D. C-Reactive Protein levels and periodontal diseases during pregnancy in malaysian women. Oral Health Prev Dent. 2018; 16(3): 281-9.

7. Tang Y, Fung E, Xu A, Yao H. C-reactive protein and ageing. Clin Exp Pharmacol Physiol. 2017; 44: 9-14.

8. Martínez V, Carrillo B, Guzmán E, Puerto M, Bermeo J, Pozos A. Proteína $C$ reactiva como marcador inflamatorio en la enfermedad periodontal. Nova Scientia, 2017; 9(2): 51-64.

9. Manzur F, Alvear C, Alayón A. Papel de la proteína C reactiva en las enfermedades cardiovasculares. Rev Colomb Cardiol 2011; 18(5): 273-8.

10. Sproston N, Ashworth J. Role of C-Reactive Protein at Sites of Inflammation and Infection. Front Immunol. 2018; 9: 754.

11. Suarez I, Reyna E, Mejia J, Reyna N, Torres D, Santos J, et al. Proteína $C$ reactiva plasmática en el segundo trimestre para predicción de parto pretérmino. Perinatología y Reproducción Humana. Elsevier. 2016; 30(2): 57-62.

12. Vecchié A, Bonaventura A, Carbone F, Maggi D, Ferraiolo A, Carloni $B$, et al. C-Reactive Protein levels at the midpregnancy can predict gestational complications. BioMed Research International. 2018; (3): 1-8.

13. Ali Z, Bokhari F, Zaki S, Zargham U, Tauseef A, Khakan S. Correlation of CRP levels in third trimester with fetal birth weight in preeclamptic and normotensive pregnant women. J Coll Physicians Surg Pak. 2015; 25(2): 111-4.

14. Rout R, Mahalik M. Comparison of C-reactive proteins level in gestational hypertension and in normal pregnancy in 2 nd and 3rd trimester and its correlation with maternal and foetal outcome. Int J Reprod Contracept Obstet Gynecol. 2019; 8(6): 2541-8.

15. Wu M, Chen S, Jiang S. Relationship between Gingival Inflammation and Pregnancy. Mediators Inflamm. 2015; 2015: 623427.

16. Zhong Z, Fu H. Effects of SPR periodontal basic therapy on cervical crevicular fluid C-reactive protein in patients with chronic periodontitis. Shanghai Kou Qiang Yi Xue. 2018; 27(5): 530-4.

17. Bansal T, Dhruvakumar D, Pandey A. Comparative evaluation of C-reactive protein in peripheral blood of patients with healthy gingiva, gingivitis and chronic periodontitis: A clinical and particle-enhanced turbidimetric immuno-analysis. J Indian Soc Periodontol. 2014; 18(6): 739-43.

18. Goyal L, Bey A, Gupta N, Sharma V. Comparative evaluation of serum C-reactive protein levels in chronic and aggressive periodontitis patients and association with periodontal disease severity. Contemp Clin Dent. 2014; 5(4): 484-8.

19. Aoyama N, Suzuki JI, Kobayashi N, Hanatani T, Ashigaki N, Yoshida 
A, et al. Associations among tooth loss, systemic inflammation and antibody titers to periodontal pathogens in Japanese patients with cardiovascular disease. J Periodontal Res. 2018; 53(1): 117-22.

20. Sarmiento LR, Velosa J, Arango D, Villegas M, Latorre C, Escobar F. Relación entre la presencia de enfermedad periodontal y el infarto agudo al miocardio por medio de la proteína $C$ reactiva ultrasensible: primera parte. Univ Odontol. 2015; 34(73): 139-48.

21. Becerra TB, Calla-Poma RD, Requena-Mendizabal MF. Antibacterial effect of peruvian propolis collected during different seasons on the growth of Streptococcus Mutans. Open Denstistry J. 2019; 13(1): 327-31.

22. Huang W, Wu J, Mao Y, Zhu S, Huang GF, Petritis B, et al. Developing a periodontal disease antibody array for the prediction of severe periodontal disease using machine learning classifiers. J Periodontol. 2020; 91(2): 232-43.

23. Musalaiah S, Anupama M, Nagasree M, Krishna CM, Kumar A, Kumar PM. Evaluation of nonsurgical periodontal therapy in chronic periodontitis patients with anemia by estimating hematological parameters and high-sensitivity C-reactive protein levels. J Pharm Bioallied Sci. 2014; 6(Suppl. 1): S64-9.

24. Atta J, Ahmed Z, Aslam N, Nawaz H, Das T, Ali Z. C-reactive protein in patients with preeclampsia. Indo Am J Pharm Sci. 2017; 4(3).

25. Peña E, Reyna E, Mejia J, Reyna N, Torres D, Santos J. Proteína C Reactiva en pacientes con preeclampsia y gestantes normotensas sanas. Clin Invest Gin Obst. 2016; 43(1): 7-11.

26. Vecchié A, Bonaventura A, Carbone F, Maggi D, Ferraiolo A, Carloni B, et al. C-Reactive Protein Levels at the Midpregnancy Can Predict Gestational Complications. Biomed Res Int. 2018.

27. The World Bank. World Development Indicators [Internet]. 2020. Disponible en: https://databank.worldbank.org/reports. aspx? source=2\&series=SH.DYN.NMRT\&country=

28. Araya C, Galindo MP. Tamaño de la muestra en investigación clínica. Med Clin (Barc). 2009; 133(1): 26-30.

29. Bascones A. Periodoncia clínica e implantología oral. Ediciones Avances. Barcelona. 2009.

30. Química Clínica Aplicada. Látex PCR directo. Test en placa para la determinación in vitro de la Proteína C Reactiva. 2009.

31. Ministerio de Salud del Perú. Estrategia Sanitaria Nacional De Salud Bucal [Internet]. 2014. Disponible en: https://www.minsa. gob.pe/portalweb/06prevencion/prevencion_2.asp?sub5=13

32. Mannava P, Gokhale S, Pujari S, Biswas KP, Kaliappan S, Vijapure S. comparative evaluation of C-reactive Proteins in pregnant women with and without periodontal pathologies: a prospective cohort analysis. J Contemp Dent Pract. 2016; 17(6): 480-3.
33. Wyczalkowska A, Czarkowska B, Zielenkiewicz M, Paczek L. Inflammatory markers change with age, but do not fall beyond reported normal ranges. Arch Immunol Ther Exp. 2016; 64: 249-54.

34. Opeodu I, Dosumu E, Arowojolu M. periodontal condition and treatment needs of some pregnant women in Ibadan, Nigeria. Ann Med Health Sci Research. 2015; 5(3): 213-7.

35. Kashetty M, Kumbhar S, Patil S, Patil P. Oral hygiene status, gingival status, periodontal status, and treatment needs among pregnant and nonpregnant women: a comparative study. J Indian Soc Periodontol. 2018; 22(2): 164-70.

36. Lasserre J, Brecx M, Toma S. Oral microbes, biofilms and their role in periodontal and peri-implant diseases. Materials (Basel). 2018; 11(10): 1802.

37. Vieira A, Magalhães C, Hartenbach F, Martins do Souto R, Maciel da Silva C. Periodontal-disease-associated biofilm: A reservoir for pathogens of medical importance. Microb Pathog. 2016; 94: 27-34.

\section{Correspondencia:}

Pablo Alejandro Millones Gomez

Dirección: Avenida Cesar Vallejo 1429, Lima, Perú.

Teléfono: 944908425

Correo electrónico: pablodent@hotmail.com

Recibido: 23 de enero de 2020

Evaluado: 27 de abril de 2020

Aprobado: 12 de mayo de 2020

( ) La revista. Publicado por Universidad de San Martín de Porres, Perú. (cc) Br Licencia de Creative Commons Artículo en acceso abierto bajo términos de Licencia Creative Commons Atribución 4.0 Internacional. (http://creativecommons.org/licenses/by/4.0/)

ORCID iDs

Sandra Jessenia Pesantes Sangay $\odot$ https://orcid.org/0000-0002-3841-2735

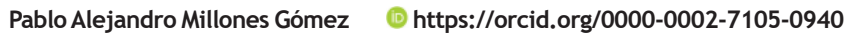

